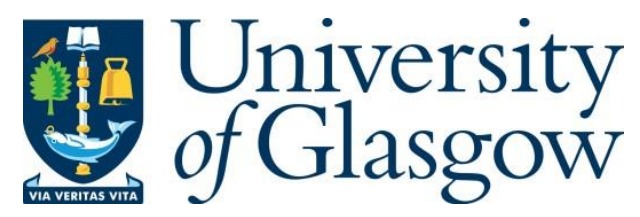

Zhang, Y., Bauer, R., Whitmer, W. M., Jackson, J. C., Windmill, J. F.C. and Uttamchandani, D. (2018) A MEMS Microphone Inspired by Ormia for Spatial Sound Detection. In: 2018 IEEE Micro Electro Mechanical Systems (MEMS), Belfast, UK, 2125 Jan 2018, pp. 253-256. ISBN 9781538647820.

There may be differences between this version and the published version. You are advised to consult the publisher's version if you wish to cite from it.

http://eprints.gla.ac.uk/226330/

Deposited on: 23 November 2020

Enlighten - Research publications by members of the University of Glasgow http://eprints.gla.ac.uk 


\title{
A MEMS MICROPHONE INSPIRED BY ORMIA FOR SPATIAL SOUND DETECTION
}

\author{
Yansheng Zhang ${ }^{1}$, Ralf Bauer ${ }^{1}$, William M. Whitmer ${ }^{2}$, Joseph C. Jackson ${ }^{1}$, James F.C. Windmill, \\ and Deepak Uttamchandani ${ }^{1}$ \\ ${ }^{1}$ University of Strathclyde, Glasgow, UK \\ ${ }^{2} \mathrm{MRC} / \mathrm{CSO}$ Institute of Hearing Research, Glasgow, UK
}

\begin{abstract}
This work introduces a MEMS microphone with two pairs of orthogonal and joined sensor membranes, with independent acoustic directionality responses, leading to a 3D sound localization potential. This single microphone can thus be regarded as two individual bi-directional microphones. Combining this architecture with the fly Ormia ochracea's tympana mechanism, this microphone is also the first biomimetic MEMS microphone with piezoelectric sensing, designed for 2D sound localization.
\end{abstract}

\section{INTRODUCTION}

MEMS directional microphones inspired by the hearing mechanism of the parasitoid fly Ormia ochracea have been regularly reported in multiple variations recently. One of the well-known designs was developed by Miles et al. [1,2], consisting of two identical connected diaphragms. They rotate along the common hinge that mimics the mechanical coupling between Ormia's two tympana when locating a cricket host. Recently, Kuntzman et al. [3] added piezoelectric sensing to this kind of design allowing it to be used in some critical environment such as high humidity. Compared to the traditional MEMS directional microphones designs that have multiple sound access holes in their package to generate sound pressure gradients through a carefully designed sound path, the Ormia-inspired microphones introduced above only require one surface to be exposed to the medium, which is more preferable for packaging considerations [4]. In addition, it has been proved having higher SNR than commercial microphones currently available [5].

So far, most previous studies have only focused on one dimensional (1D) (i.e. azimuth plane) sound localization [1 $-3,6]$, which have constraints for being applied as independent spatial sound localization sensors in applications such as auditory scene analysis for hearing aids or the determination of multiple sound sources in a crowded environment (e.g. battlefield, cocktail party, etc.). Currently, combination of arrays of well-distributed microphones with various sound localizing algorithms are implemented for these purposes [7]. The microphone array used can be comprised of a number of identical omnidirectional microphones separated in a three dimensional (3D) space. It can also be a pair of orthogonally oriented or three triangular distributed bi-directional microphones. Comparing these two types of microphone array, although the omni-directional array provides a more accurate sound location than a pair of bi-directional microphones in most cases, the size friendly bi-directional microphone array appeals more to small applications. Next to sound localizing applications, two orthogonally placed bidirectional microphones also are frequently used as stereo recording technique [8] (i.e. Blumlein pair) to produce high

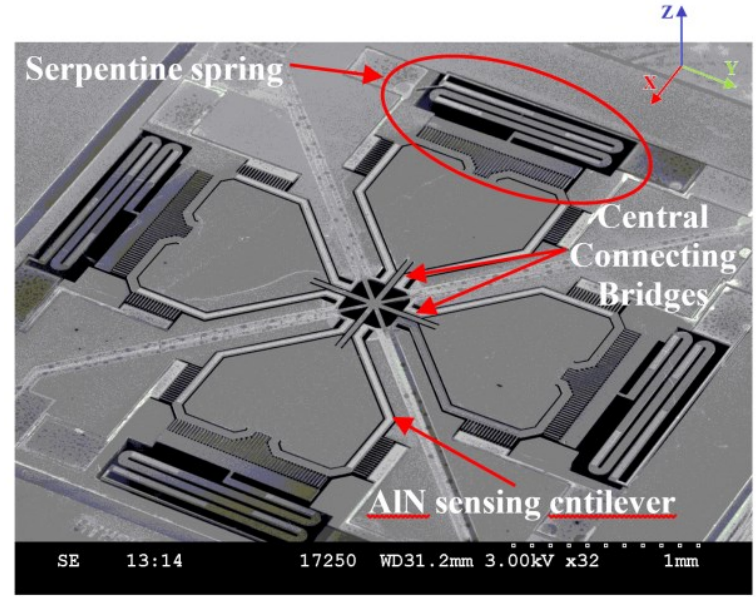

Figure 1: Image of the device taken by Scanning Electron Microscope (SEM)

quality stereo images when the microphones are placed in the short distance to the sound source and in low noise environment. However, due to the mismatch and the physical separation between each microphone in an array, further data processing is required to compensate for any errors.

The aim of the presented research is to design an Ormia-inspired MEMS directional microphone for 2D sound localization. It has two pairs of orthogonally oriented and joined sensor membranes, with the directional patterns of each pair of membranes independent of each other. This single microphone can thus be regarded as two individual spatial bi-directional microphones.

\section{DESIGN AND SIMULATION}

The device is manufactured by using the commercial PiezoMUMPs process, a multi-user MEMS foundry service designed for piezoelectric devices and provided by MEMSCAP Inc. It consists of four heart-shaped single crystal silicon membranes with $10 \mu \mathrm{m}$ thickness, connected by two $800 \mu \mathrm{m} \times 30 \mu \mathrm{m}$ rectangular beams acting as two bridges in the center as shown in Figure 1. Additionally, another two $200 \mu \mathrm{m} \times 30 \mu \mathrm{m}$ beams also cross at the center, and are fixed to the substrate in order to support the vibrational membranes. Each heart-shaped membrane is allocated two circumferential cantilevers coated with a piezoelectric AIN sensing layer, with one end fixed to the substrate and the other end connected to the wide end of the membrane. Furthermore, each membrane has three sets of flexible comb fingers (one set located at the end of the membrane and two sets at the sides) with a $5 \mu \mathrm{m}$ gap between each fingers for future capacitive sensing 
development. To ensure that at the initial state the fixed comb fingers well overlap with the flexible fingers at the end of the membrane, which has high initial curvature due to intrinsic stress, a serpentine cantilever with designed surface stresses has been added to support the fixed comb fingers. Each serpentine spring has several AlN and metal layers deposited at specified locations to generate sufficient initial curvature to match the curvature of the membranes. The addition of the comb fingers also introduces further air damping in the slits between the membranes and the substrate aiming to expand the working frequency range. The device encloses a $400 \mu \mathrm{m}$ thick air cavity underneath the released membranes. Ignoring the fixed capacitive fingers and serpentine springs, the active region is about $2.6 \mathrm{~mm} \times 2.6 \mathrm{~mm}$.

Previous studies [9] on the mechanism of Ormia's hearing organ reveals that the vibration displacement of its two tympana has a sine dependence on the sound zenith angle $\theta$ (see Figure 2 for direction definition) around the frequency of its host's mating calls which overlaps with the first resonance frequency of the tympana. Therefore, a microphone structure with two membranes and a connecting bridge that mimics Ormia's tympana and its interaural tissue bridge can also provide similar directional response to the sound zenith angle. When two pairs of membranes are placed orthogonally and centrally connected, the directional response behavior is not influenced by the presence of the connection. Each pair of opposite membranes has a figure- 8 directional polar pattern with respect to the zenith incident angle $\theta$ as shown in Figure 2 (b) and (c). Next to this, the two pairs of membranes also perform as two bi-directional microphones in the azimuth plane. The simulated results in Figure 2 (a) illustrate that, if $\theta=90^{\circ}$, the maximum response of each pair of opposite membranes occurs at the azimuth angle that is parallel with its own longitudinal axis, and the minimum response is generated when the sound azimuth angle is parallel with the longitudinal axis of

(a)

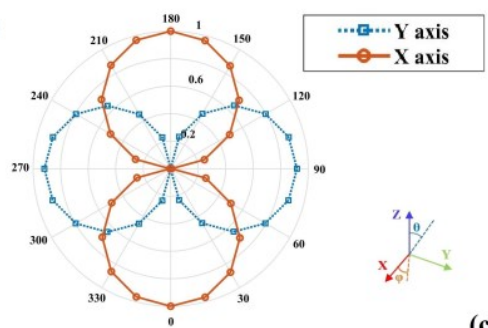

(b)
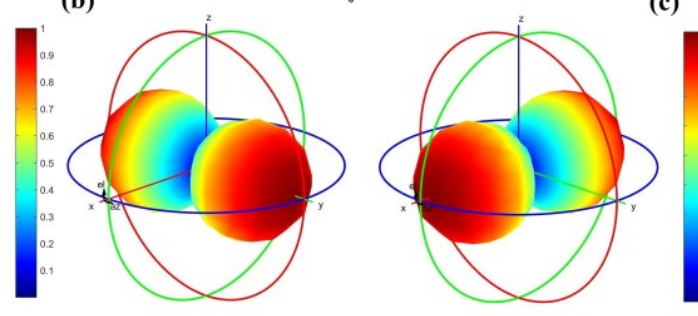

Figure 2: Simulated directional polar patterns of: (a) Two pairs of membranes in XY plane (i.e. $\varphi$ changes from 0 to 360 degrees); (b) One pair of membranes where the longitudinal axis is parallel with Y axis; (c) The other pair of membranes where the longitudinal axis is parallel with $X$ axis.
Simulated:
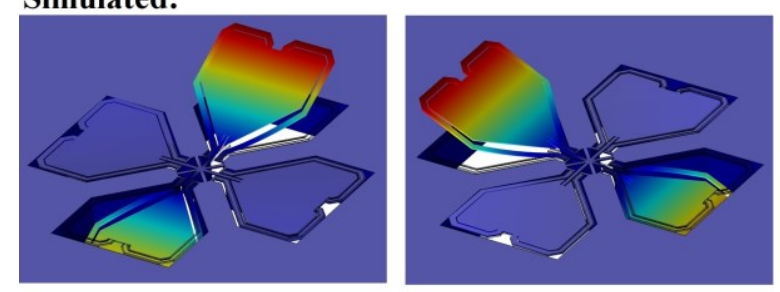

Measured:
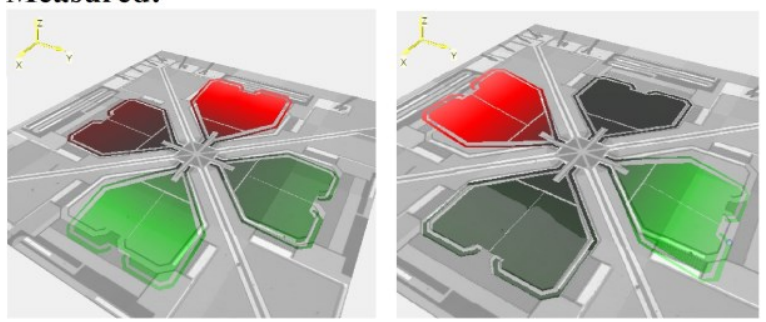

Figure 3: Simulated and measured (by 3D LDV) rocking mode shapes of the device at around the $1^{\text {st }}$ resonance frequency $(2.8 \mathrm{kHz})$

another pair of membranes.

\section{EXPERIMENTAL RESULTS}

The mechanical performance of the prototype was characterized using a Polytec MSA-100-3D scanning vibrometer. A Visaton round loud speaker was placed 40 $\mathrm{cm}$ away from the center of the scanning stage on which the prototype was fixed. The acoustic excitation signal consisted of a chirp signals with a bandwidth of $10 \mathrm{kHz}$ and the sound azimuth and zenith incident angles are changed via positioning of the speaker. A Brüel \& Kjær (B\&K) 4138 microphone was placed close to the scanning stage, acting as a reference microphone. The measured eigenmodes of Figure 3 show that every pair of opposite membranes vibrate out of phase at the first resonance frequency $-2.8 \mathrm{kHz}$, similar to the first mode shape of Ormia's tympana, closely matched with the simulated mode shapes obtained from finite element modeling.

Since the geometry of this MEMS microphone is symmetric both in $\mathrm{X}$ and $\mathrm{Y}$ direction, the displacement amplitude at point 1 and point 2 approximately equals the one at their opposite positions in the same pair of membranes, albeit at a $180^{\circ}$ phase shift in both cases. Therefore, only the displacement of two points (point 1 and point 2 in Figure 4(a)) was extracted to analyze the mechanical response of the two orthogonal membranes. This experimental method was also used for measuring the electrical output as covered later. When $\theta=45^{\circ}$ and the sound incidents along the longitudinal axis of the pair of membranes including point 1 , point 1 has the maximum amplitude response at around $60 \mathrm{~nm} / \mathrm{Pa}$ at the first resonance frequency whereas the response taken at point 2 is just one-third of this value, and vice-versa when the sound azimuth incidence is rotated by $90^{\circ}$. Moreover, as shown in Figure 4(b), when decreasing the sound zenith angle $\theta$ from $90^{\circ}$ to $30^{\circ}$, the mechanical response of the membrane at the $2.8 \mathrm{kHz}$ is also reduced from $68 \mathrm{~nm} / \mathrm{Pa}$ to $31 \mathrm{~nm} / \mathrm{Pa}$, proving its directivity in the zenith plane. The same directionality is also verified by the two similar acquired mechanical responses from the orthogonal 
(a)

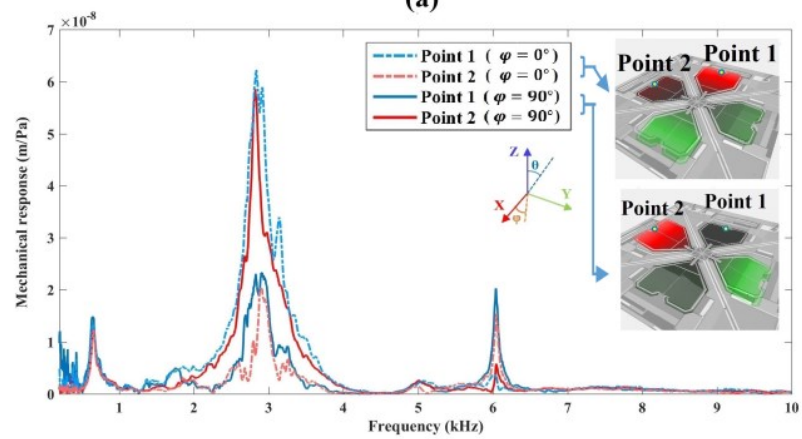

(b)

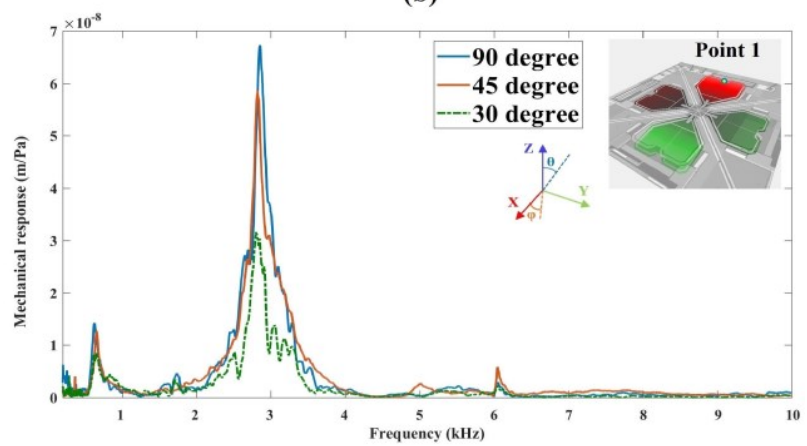

Figure 4: Mechanical response of the device with sound incident at: (a) $\varphi=0^{\circ}$ and $90^{\circ}, \theta=45^{\circ}$ at point 1 and point 2 , respectively. The maximum value at a certain zenith angle appears when the sound azimuth angle is parallel with the longitudinal axis of any pair of membranes; (b) $\varphi=90^{\circ}, \theta=0^{\circ}, 45^{\circ}$ and $90^{\circ}$ at point 1.

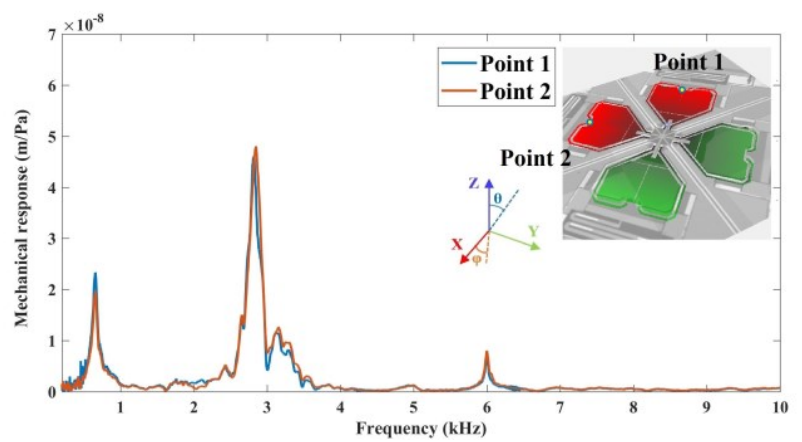

Figure 5: Mechanical response of the device at points land 2 when sound is incident at $\varphi=45^{\circ}$ and $\theta=45^{\circ}$, respectively.

membranes when both $\varphi$ and $\theta$ equal to $45^{\circ}$, showing the nearly identical mechanical response of point 1 and point 2 at $2.8 \mathrm{kHz}$ (see Figure 5). The noise and the peak below 1 $\mathrm{kHz}$ are mainly due to the shaking of the reference microphone when the scanning stage alters the position in order to change the laser focusing point. The second resonance frequency of each membrane pair is measured around $6.1 \mathrm{kHz}$, corresponding to a twisting mode shape where a single membrane twists along its central connecting bridge. However, this twisting response can be compensated by summing the electrical output signals acquired at two opposite sides of the head-shaped using either piezoelectric sensing or capacitive sensing.

For the electrical output measurement using piezoelectric sensing, the experimental setup includes a
ESS Heil Air-motion Transformer located $40 \mathrm{~cm}$ from the prototype as acoustic source, with MEMS that was wirebonded onto a $1 \mathrm{~mm} \times 1 \mathrm{~mm}$ custom PCB. The prototype $\mathrm{PCB}$ is mounted on a rotary stage in with the front surface of the MEMS is directly facing the sound source. Similar to the setup of measuring its mechanical behaviors, the same B\&K microphone was set beside the device and works as a reference as well. The output port of the device was directly connected to an oscilloscope using BNC cable and the displayed output data was collected by a custom LabVIEW program.

As shown in Figure 6, when $\varphi=0^{\circ}$ (i.e. a sound is incident from the wide end of the heart-shaped membrane opposite to port 1) the piezoelectric voltage generated at port 1 approaches $0.29 \mathrm{mV} / \mathrm{Pa}$ around $3 \mathrm{kHz}$ while the acoustic response at port 2 is just about $0.11 \mathrm{mV} / \mathrm{Pa}$. The ratio of acoustic response $(\approx 2.6)$ at these two points at the first resonance frequency is close to the ratio of mechanical response shown in Figure 4. As the piezoelectric material is attached onto the cantilevers on the side of the membrane, it also generates significant charge when the membrane twists, which explains high response shown around the $6 \mathrm{kHz}$ movement at the second resonance mode.

To further investigate the directivity of the device, a measurement of spatial directional polar patterns at $3 \mathrm{kHz}$ was taken at port 1 and port 2. Only one-eighth of the 3D space was considered according to the full symmetric behavior demonstrated before. The measured directional polar patterns of the two pairs of membranes in a oneeighth 3D space are shown in Figure 7 and Figure 8. Although the acoustic response generated at port 1 and port 2 has an offset when the exciting sound wave vertically hits

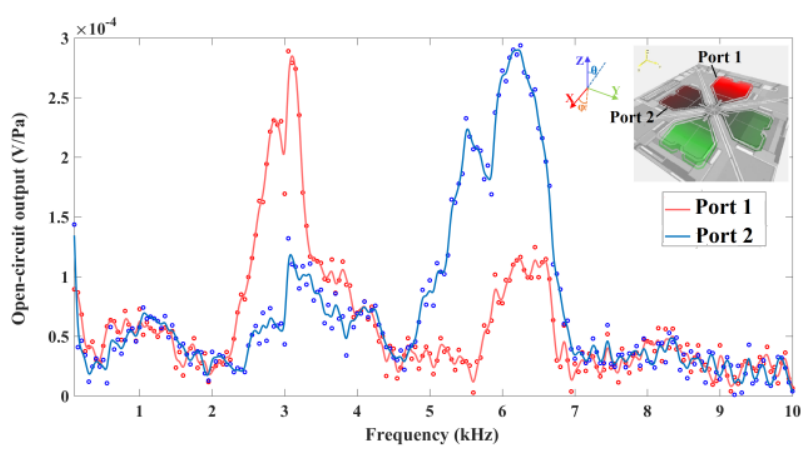

Figure 6: Open-circuit acoustic response acquired from port 1 and port 2 using piezoelectric sensing when sound is incident at $\varphi=0^{\circ}$ and $\theta=90^{\circ}$.

the front surface of the device or propagates along the central rotating hinge of each pair of membranes, the directional polar patterns in the azimuth plane of the two pairs of membranes are still orthogonal with each other as illustrated in Figure 7. In addition, the directional behavior of two pairs of membranes also exists in the zenith plane as expected from simulations (see Figure 8). Combining the results of these two figures, it is clear that the device can be regarded as two individual orthogonally oriented directional microphones providing spatial bi-directional polar patterns but jointed into a single MEMS device.

\section{DISCUSSION AND CONCLUSION}

The MEMS microphone presented is the first 


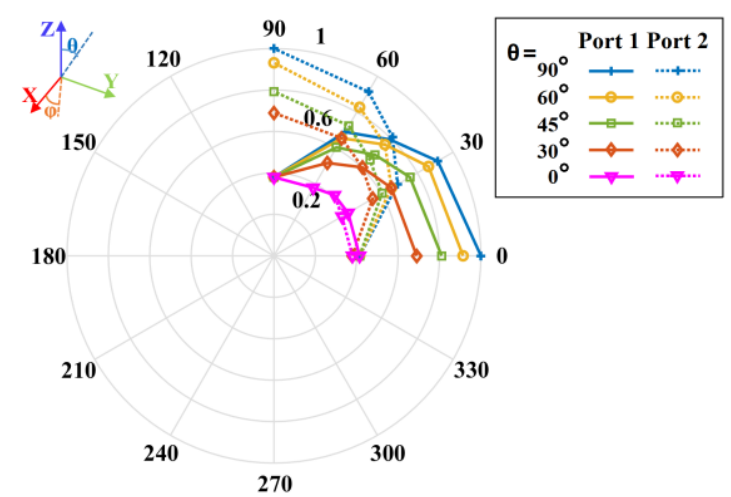

Figure 7: Directional polar patterns obtained at port 1 and port 2 when sound incident angle $\varphi$ increase from $0^{\circ}$ to $90^{\circ}$ in a one-eighth $3 D$ space. The polar coordinate is parallel with the XY plane (i.e. azimuth plane).

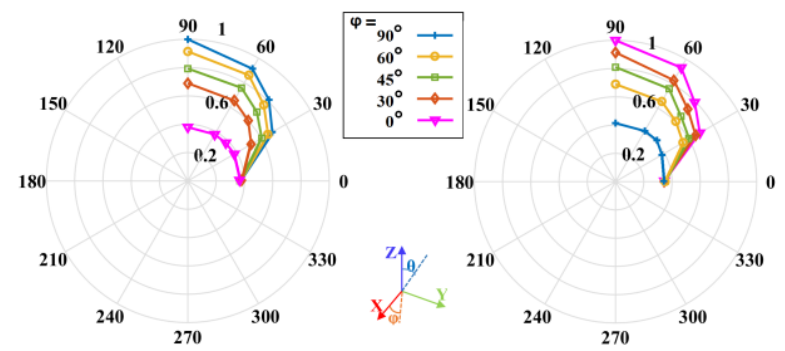

Figure 8: Directional polar patterns obtained at port 1 (left) and port 2 (right) when sound incident angle $\theta$ increase from $0^{\circ}$ to $90^{\circ}$ in a one-eighth $3 D$ space. For Port 1 , the polar coordinate is parallel with XZ plane. For Port 2, the polar coordinate is parallel with the YZ plane.

generation prototype inspired by the combination of a bidirectional microphone array and the hearing mechanism of Ormia ochracea and is designed for sound localization in a 3D space. Both mechanical and electrical experimental results prove that the presented device has a directional behaviour similar to an array of two orthogonally placed bi-directional microphones at the first resonance frequency around $3 \mathrm{kHz}$, and achieves the basic concept. As there are only two resonance mode shapes below $10 \mathrm{kHz}$ with the second mode shape representing a twisting movement that can be electronically compensated for, theoretically, the microphone should have the same directional behaviour across the frequencies below $10 \mathrm{kHz}$, which is planned to be demonstrated in future work.

Since the surface area of piezoelectric material is much less than in other previous designs, the acoustic response under rocking movement is about 10 times lower than in Ormia-inspired devices previous demonstrated, which could be improved in the future designs. A customized readout circuit will be developed for the piezoelectric sensing unit of this specific design to allow low-noise differential output for investigating the directivity of the device in the entire 3D space in the future. Moreover, characterization of the capacitive sensing potential and comparing the acoustic response with the one taken using the piezoelectric sensing method are the next critical tasks.
This work was supported by the EPSRC under grant EP/M026701/1 and by the European Research Council under the European Union's Seventh Framework Programme (FP/2007-2013) / ERC Grant Agreement n. [615030].

\section{REFERENCES}

[1] R. N. Miles, et al., "A low-noise differential microphone inspired by the ears of the parasitoid fly Ormia ochracea", J. Acoust. Soc. Am., vol. 125, pp. 2013-2026, 2009.

[2] R. N. Miles, et al., "A MEMS Low-noise Sound Pressure Gradient Microphone With Capacitive Sensing", J. Microelectromech. Syst., vol. 24, no. 1, 2015.

[3] M. L. Kuntzman, et al., "Micromachined In-Plane Pressure Gradient Piezoelectric Microphones", IEEE Sensors J., vol. 15, no. 3, pp. 1347-1357, 2015.

[4] R. Bauer, et al., "Influence of microphone housing on the directional response of piezoelectric MEMS microphones inspired by Ormia ochracea", IEEE Sensors J., vol. 17, no. 17, pp. 5529-5536, 2017

[5] R. N. Miles, "Comparisons of the performance of Knowles hearing aid microphones to that of the Binghamton Ormia-inspired gradient microphone," State Univ. of New York at Binghamton, Binghamton, NY, Sep. 13, 2015

[6] H. J. Liu, M. Yu, and X. M. Zhang, "Biomimetic optical directional microphone with structurally coupled diaphragms," Appl. Phys. Lett., vol. 93, no. 24, p. 243902, Dec. 2008.

[7] S. Mohan et al., "Localization of multiple acoustic sources with small arrays using a coherence test", J Acoust Soc Am., vol 123, no. 4, 2008

[8] A. D. Blumlein, "Improvements in and relating to Sound-transmission, Sound-recoring and Soundreproducing Systems," U.K. Patent 394325, June. 14, 1933

[9] R. N. Miles, D. Robert, and R. R. Hoy, "Mechanically coupled ears for directional hearing in the parasitoid fly Ormia ochracea.," J. Acoust. Soc. Am., vol. 98, no. 6, pp. 3059-3070, 1995.

\section{CONTACT}

*Y. Zhang, yansheng.zhang.101@strath.ac.uk 